\title{
A two-parameter discrete distribution with a bathtub hazard shape
}

\author{
Ammar M. Sarhan ${ }^{1, a}$ \\ ${ }^{a}$ Department of Mathematics and Statistics, Dalhousie University, Canada
}

\begin{abstract}
This paper introduces a two-parameter discrete distribution based on a continuous two-parameter bathtub distribution. It is the only two-parameter discrete distribution that shows a bathtub-shaped hazard function. Some statistical properties of the distribution are discussed. Three different methods are used to estimate its two unknown parameters. The point estimators of the parameters have no closed form. The bootstrap method is used to estimate the distributions of these point estimators. Different approximations of the interval estimations for the two-parameters are discussed. Real data sets are analyzed to show how this distribution works in practice. A simulation study is performed to investigate the properties of the estimations obtained and compare their performances.
\end{abstract}

Keywords: maximum likelihood estimator, likelihood interval, least square, quartile, reliability, lifetime models

\section{Introduction}

In the fields of life testing experiments and reliability analysis, much emphasis has been given to modeling through continuous lifetime distributions, see for example Gnedenko and Ushakov (1995), Kapur and Lamberson (1977), Lawless (1982), and Sinha (1986). However, it is sometimes inconvenient or impossible to measure the lifetime of a device on a continuous scale. In many practical situations, the lifetime of a device/human is considered a discrete random variable. As examples of discrete random variables are the number of voltage fluctuations which an electronic unit can withstand before failure, the number of hours/days/weeks of hospital stay, the lifetime of the switch in the case of an on/off-switching unit and the number of cycles prior to failure of equipment operating in cycles. Even for a continuous operation, involving a continuous measurement of lifetime, observations made at periodic time points produce a discrete situation and discrete model might be more appropriate.

In the past, the geometric and negative binomial distributions are known to be discrete alternatives for exponential and gamma distributions, respectively. Three versions of discrete Weibull distribution are introduced by Nakagawa and Osaki (1975), Padgett and Spurrier (1985), and Stein and Dattero (1984) as the discrete counterpart of the usual continuous Weibull distribution.

Recently, Roy $(2003,2004)$ introduced discrete normal and discrete Rayleigh distributions, respectively, using the discretization of the continuous distribution approach. Discrete Burr type II is introduced by Al-Huniti and Al-Dayian (2012), using the same approach. Nooghabi et al. (2012)

\footnotetext{
${ }^{1}$ Department of Mathematics and Statistics, Dalhousie University, 6299 South St, Halifax, NS B3H 3J5, Canada.

E-mail: ammar.sarhan@dal.ca
}

Published 31 January 2017 / journal homepage: http://csam.or.kr

(c) 2017 The Korean Statistical Society, and Korean International Statistical Society. All rights reserved. 
used the continuous modified Weibull distribution of Lai et al. (2003) to propose a new modified version of discrete Weibull distribution (DMW). Bebbington et al. (2012) introduced discrete additive Weibull distribution (DAddW) using the continuous additive Weibull distribution of Xie and Lai (1996). Recently, Almalki and Nadarajah (2014) presented a new discrete modified Weibull (NMWD) distribution as a discrete analogue of a recent modified continuous Weibull distribution by Almalki (2013).

The hazard rate function of most of these distributions is monotonic. Therefore, they cannot be as good of fit for real data sets that exhibit a non-monotonic hazard function. Most of real data sets have a bathtub-shaped hazard. Curretnly there are only three discrete distributions (DMW, DAddW and NDMW with three, five and three parameters, respectively) that show a bathtub-shaped hazard function. Models with less parameter numbers are preferable for different reasons, such as ease in simulating draws from and less difficulty in estimating model parameters. This motivates the reason for looking for models with a fewer parameters that accommodate different shapes of hazard function.

Chen (2000) revisited a two-parameter bathtub distribution we refer to as TPBT. Chen (2000) addressed that the hazard function of the TPBT can be either increasing or bathtub-shaped. The TPBT distribution does not have a scale parameter; however, it has good properties. The confidence intervals and the joint confidence regions for the two-parameters have a closed form (Chen, 2000). There are no existing two-parameter distributions with bathtub failure rates that have exact joint confidence regions for parameters (Chen, 2000). Furthermore, this model is used as suitable fit for different lifetime data sets. These good features of the TPBT model encourage me to discuss its discrete analogue.

In this paper, we use the TPBT distribution as a continuous lifetime model and follow the discretization approach to introduce its discrete analogue. We refer to it as the discrete two-parameter bathtub (DTPBT) distribution. The hazard rate function of the DTPBT distribution can be either increasing or bathtub-shaped. Therefore, it can be used to fit various real data sets. It will be the only two-parameter discrete distribution which shows a bathtub hazard shape.

The rest of the paper is organized as follows. In Section 2 we give a brief description of the continuous two-parameter bathtub (TPBT) distribution and introduce the DTPBT distribution. We also discuss some of its characteristics. In Section 3, we use the percentile, the least square and the maximum likelihood methods to estimate its two unknown parameters. Three different approximations to the confidence intervals of the two-parameters are discussed in Section 4. In Section 5, real data sets are analyzed using new distribution to compare it with DW, DMW, DAddW, and DRMW distribution. A simulation study is conducted in Section 6. Finally, Section 7 concludes the paper and discusses some future work.

\section{The DTPBT distribution}

\subsection{Continuous TPBT distribution}

Chen (2000) revisited a two-parameter continuous distribution with a bathtub-shaped hazard function as well as studied some of its characteristics. The survival function (sf) of this distribution is

$$
S(t ; \alpha, \beta)=\exp \left\{-\alpha\left(e^{t^{\beta}}-1\right)\right\}, \quad \alpha, \beta>0, t \geq 0 .
$$

Here $\alpha$ and $\beta$ are both shape parameters. The hazard function (hf) of this distribution is

$$
h(t ; \alpha, \beta)=\alpha \beta t^{\beta-1} e^{t^{\beta}} .
$$

It is easy to check hf by looking at the sign of the first derivative of $h(t), h^{\prime}(t)=\alpha \beta t^{\beta-2} e^{t^{\beta}}\left(\beta-1+\beta t^{\beta}\right)$, which shows that $h^{\prime}<0$ when $t<(1 / \beta-1)^{1 / \beta}$. That is, the hazard function $h(x)$ can be: (i) increasing 
when $\beta \geq 1$; (ii) of bathtub shape when $\beta<1$.

\subsection{The discrete analogue}

Using the continuous TPBT distribution and by discretization, we propose the DTPBT distribution. Let $T$ be a real positive random variable describing a lifetime of a devise that follows TPBT distribution. Let $X=\lfloor T\rfloor+1$, where $\lfloor T\rfloor$ is the floor function, then $X$ is a random variable that follows DTPBT distribution. The sf of DTPBT distribution can be obtained from (2.1) by setting $q=e^{-\alpha} \in(0,1)$, as

$$
S(x)=P(X>x)=q^{e^{x^{\beta}}-1}=\frac{r(x)}{q}, \quad x=1,2, \ldots ; \beta>0 .
$$

The cumulative distribution function (cdf) is

$$
F(x)=P(X \leq x)=1-S(x), \quad x=1,2, \ldots ; \beta>0 .
$$

The probability mass function (pmf) is

$$
p(x)=P(X=x)=\frac{1}{q}[r(x-1)-r(x)]=\frac{1}{q} w(x), \quad x=1,2, \ldots ; \beta>0 .
$$

The hazard rate function (hrf) is, for $r(x)=q^{\exp \left(x^{\beta}\right)}$,

$$
h(x)=P(X=x \mid X \geq x)=\frac{P(X=x)}{P(X>x-1)}=1-\frac{r(x)}{r(x-1)} .
$$

It is interesting to note that $h(x) \leq 1$ in discrete time. To study the behavior of the hazard function, we define $\beta_{0}=\ln (2 e-1) / \ln 2 \simeq 0.5752$ and define $k(x, \beta)$ as a function of $x$ and $\beta$ as

$$
k(x, \beta)=2 e^{x^{\beta}}-e^{(x-1)^{\beta}}-e^{(x+1)^{\beta}}, \quad \text { for } x=1,2, \ldots
$$

Note that $\beta_{0}$ is the solution of $k(1, \beta)=0$ in $\beta$.

Proposition 1. (i) For a fixed value of $\beta, k(x, \beta)$ is a decreasing function in $x$, (ii) if $\beta>\beta_{0}, k(x, \beta)<$ 0 , for $x=1,2, \ldots$, (iii) if $\beta<\beta_{0}, k(x, \beta)$ is a concave function in $\beta$, for $x=2,3, \ldots$.

Figures 1 and 2 plot $k(x, \beta)$ versus $x$ at different $\beta$ and versus $\beta$ at different values of $x$, respectively. From these plots, one can see the behavior of $k(x, \beta)$.

Theorem 1. Let $X$ be a DTPBT distributed random variable with parameters $q$ and $\beta$. Then the hrf of $X$ : (i) is increasing if $\beta>0.5752$, (ii) takes a bathtub shape when $\beta<0.5752$.

Proof: Let $\Delta h=h(x+1)-h(x)$, for $x=1,2, \ldots$. It is obvious that: (i) if $\Delta h<0$, the hrf will be decreasing; (ii) if $\Delta h>0$, the hrf will be increasing; (iii) if $\Delta h=0$, the hrf will be flat. Using (2.6) and (2.7), we can express $h(x)$ as

$$
\Delta h=\frac{r(x+1)}{r(x)}\left[q^{k(x, \beta)}-1\right],
$$

since $r(x+1) / r(x)>0, x=1,2, \ldots$, therefore the sign of $\Delta h$ depends only on $q^{k(x, \beta)}-1$. Thus, (i) if $k(x, \beta) \ln q>0$, equivalently if $k(x, \beta)<0$, then $\Delta h>0$, which leads to an increasing hrf; (i) if 


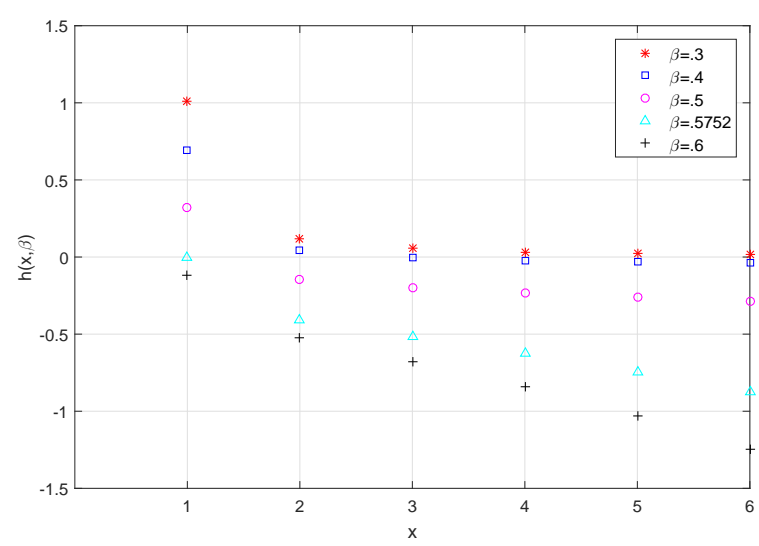

Figure 1: Plots of $k(x, \beta)$ versus $x$ at different values of $\beta$.
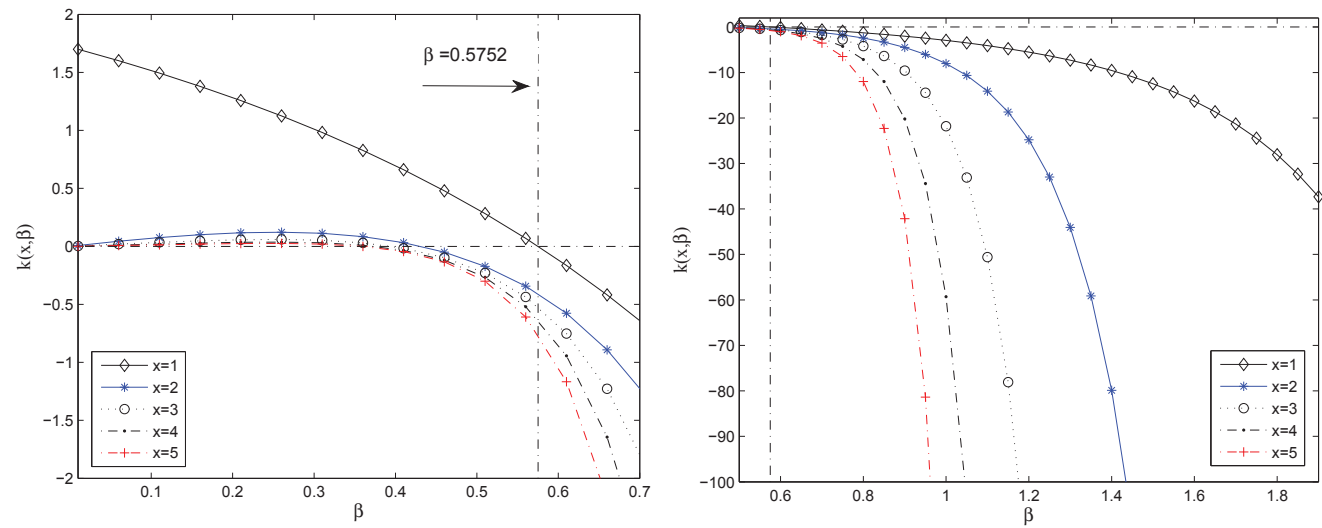

Figure 2: Plots of $k(x, \beta)$ versus $\beta$ at different values of $x$.

$k(x, \beta) \ln q<0$, equivalently if $k(x, \beta)>0$, then $\Delta h<0$, which leads to a decreasing hrf; and (iii) if $k(x, \beta) \ln q$ changes its sign from negative to positive, equivalently if the sign of $k(x, \beta)$ changes from positive to negative, then the sign of $\Delta h$ will change from negative to positive, which leads to a bathtub hrf. Using the results of Proposition 1 completes the proof.

Figure 3 shows different patterns of the hazard function when $q=0.7$ for $\beta=0.2,0.3,0.4,0.5,0.6$, and 1.5 .

- Percentiles: Using the cdf (2.4), we obtain the $100 p^{\text {th }}$ percentile, $0<p<1$, of $\operatorname{DTPBT}(q, \beta)$ as

$$
x_{p}=\left\lfloor\left\{\ln \left[1+\frac{\ln (1-p)}{\ln q}\right]\right\}^{\frac{1}{\beta}},\right.
$$

where $\lfloor. J$, is the floor function.

- Moments: The moments method to estimate its parameters cannot be used because the DTPBT distribution has no analytic forms for the moments. 

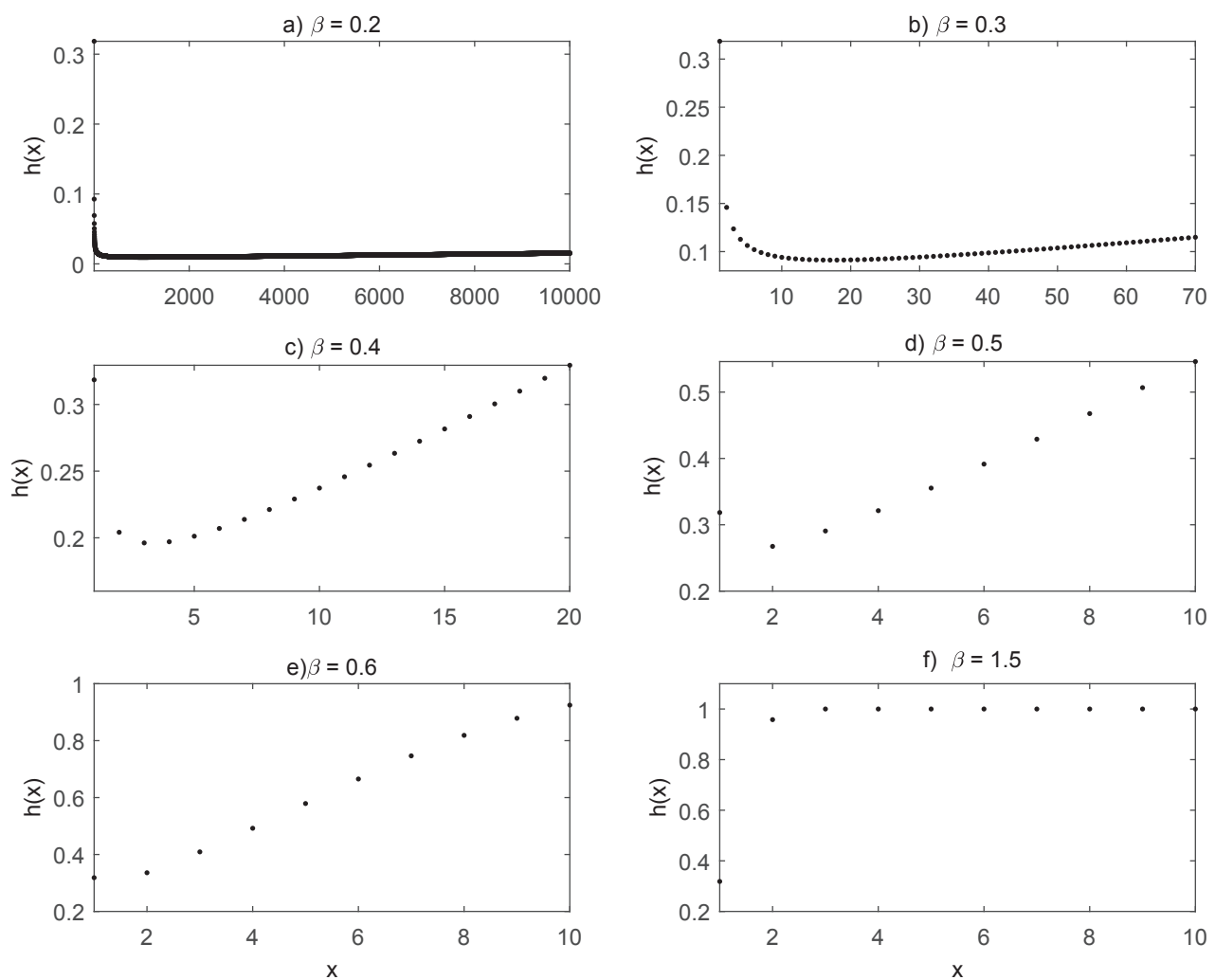

Figure 3: The hazard function at different values of $\beta$ when $q=0.7$.

\section{Parameter estimation}

In this section we discuss the estimation of two unknown parameters $\beta$ and $q$ of the DTPBT distribution based on a simple random sample $X_{1}, X_{2}, \ldots, X_{n}$ from DTPBT distribution.

\subsection{Quartile method}

Two quartiles are required because two-parameters need to be estimated. We can use the $1^{s t}$ and $3^{r d}$ quartiles. Equating data quartiles to the corresponding distribution quartiles we get a system of two equations in the two unknown parameters. Solving this system in the two unknown parameters provides the quartile estimate of the parameters.

Setting the $k^{\text {th }}$ quartile, $Q_{k}, k=1,3$, of the data to that of the distribution, we get the following system of two equations

$$
Q_{k}=\left\{\ln \left[1+\frac{\ln (1-k / 4)}{\ln q}\right]\right\}^{\frac{1}{\beta}}, \quad k=1,3 .
$$

Using (3.1), when $k=1$, we get $q$ as a function of $\beta$ as

$$
q=0.75^{\frac{1}{\exp \left(Q_{1}^{\beta}\right)-1}} .
$$


Substituting from (3.2) into (3.1), when $k=3$, we get

$$
g(\beta)=e^{Q_{1}^{\beta}}-0.2075187 e^{Q_{3}^{\beta}}-0.7924813=0 .
$$

The quartile estimate (QE) of $\beta$, say $\tilde{\beta}$, is the solution of (3.3) in $\beta$. Once we get $\tilde{\beta}$, we can use (3.2) to get the $\mathrm{QE}$ of $q, \tilde{q}$. Equation (3.3) has no analytic solution in $\beta$, therefore a numerical technique, such as a Newton-Raphson method or a graphical method, should be used. Note, it is easy to show that the quartile method fails if $Q_{1}=Q_{3}$.

\subsection{Least squares method}

The least squares (LS) method can be used to estimate the two unknown parameters $q$ and $\beta$ by minimizing the squared discrepancies between observed sf and theoretical sf, which is given as

$$
Q(q, \beta)=\sum_{i=1}^{n}\left(\hat{S}_{i}-s\left(x_{i}\right)\right)^{2}
$$

where $\hat{S}_{i}$ is the empirical sf evaluated at $x_{i}$. To do so, we set the first partial derivatives of $Q(q, \beta)$, with respect to $q$ and $\beta$, to zero, to get a system of two equations

$$
\begin{aligned}
& 0=\sum_{i=1}^{n}\left(\hat{S}_{i}-s\left(x_{i}\right)\right) s\left(x_{i}\right) x_{i}^{\beta} \ln \left(x_{i}\right) e^{x_{i}{ }^{\beta}}, \\
& 0=\sum_{i=1}^{n}\left(\hat{S}_{i}-s\left(x_{i}\right)\right) r\left(x_{i}\right)\left(e^{x_{i}{ }^{\beta}}-1\right),
\end{aligned}
$$

solving the above system in $q$ and $\beta$, gives LS estimation of $q$ and $\beta$. This system has no analytic solution. Therefore, a numerical technique should be used. The QEs can be used as initial values.

\subsection{Maximum likelihood method}

The log-likelihood function is, for $w(x)=r(x-1)-r(x)$,

$$
\mathcal{L}(q, \beta)=-n \ln q+\sum_{i=1}^{n} \ln \left[w\left(x_{i}\right)\right] .
$$

The first partial derivatives of the log-likelihood function with respect to the two-parameters are

$$
\frac{\partial L(q, \beta)}{\partial \beta}=(\ln q) \sum_{i=1}^{n} \frac{r\left(x_{i}-1\right)\left(x_{i}-1\right)^{\beta} e^{\left(x_{i}-1\right)^{\beta}} \ln \left(x_{i}-1\right)-r\left(x_{i}\right) x_{i}^{\beta} e^{x_{i}^{\beta}} \ln \left(x_{i}\right)}{w\left(x_{i}\right)},
$$

and

$$
\frac{\partial L(q, \beta)}{\partial q}=-\frac{n}{q}+\sum_{i=1}^{n} \frac{s\left(x_{i}-1\right) e^{\left(x_{i}-1\right)^{\beta}}-s\left(x_{i}\right) e^{x_{i}}}{w\left(x_{i}\right)}
$$

Setting the left side of the above two equations to zero, we get the likelihood equations as a system of two nonlinear equations in $q$ and $\beta$. Solving this system in $q$ and $\beta$ gives the maximum likelihood estimates (MLE) of $q$ and $\beta$. This system has no analytic solution, therefore a numerical method, such as Newton-Raphson method, should be used to get the MLE of the parameters. We will use package $\mathrm{R}$ or MATLAB. We can use the estimates obtained from the quartile method as initial values. 


\section{Confidence interval}

We do not have exact distributions because the point estimator of the parameters are not obtained in closed forms. Therefore, we require approximation methods because we cannot derive the exact confidence intervals of the unknown parameters. This section discusses three different approximation methods, see for example Kalbfleisch (1985) and Wasserman (2006), for the interval estimates of the two-parameters $q$ and $\beta$.

\subsection{Likelihood region and intervals}

The relative likelihood function (RLF) of the vector $\theta=(q, \beta)$ is $R(q, \beta)=L(q, \beta) / L(\hat{q}, \hat{\beta})$, where $\hat{q}$ and $\hat{\beta}$ are the MLE of $q$ and $\beta$, respectively. The $100 p \%$ likelihood region (LR) of $(q, \beta)$ is the set of parameter values $(\alpha, \beta)$ such that $R(\alpha, \beta) \geq p$, for more details we refer to Kalbfleisch (1985). For simplicity, we can take the natural logarithm of $R(\theta)$, therefore the $100 p \%$ LR of $(q, \beta)$ is the set of all values of $(q, \beta)$ which satisfy that $r(q, \beta) \geq \ln p$, where $r(q, \beta)=\ln L(q, \beta)-\ln L(\hat{q}, \hat{\beta})$. This inequality has no closed form solution. Therefore a numerical method, such as Newton-Raphson, should be used to get the likelihood intervals. Similarly, the likelihood interval (LI) for each parameter can be obtained using the maximum log-relative likelihood function of the parameter.

\subsection{Large-sample intervals}

The second partial derivatives of $\mathcal{L}$ with respect to $q$ and $\beta$ are

$$
\begin{aligned}
& \mathcal{L}_{11}=\frac{\partial^{2} \mathcal{L}}{\partial q^{2}}=-\frac{n}{q^{2}}+\sum_{i=1}^{n} \frac{w\left(x_{i}\right) w_{q q}\left(x_{i}\right)-\left[w_{q}\left(x_{i}\right)\right]^{2}}{\left[w\left(x_{i}\right)\right]^{2}}, \\
& \mathcal{L}_{12}=\frac{\partial^{2} \mathcal{L}}{\partial q \beta}=\sum_{i=1}^{n} \frac{w\left(x_{i}\right) w_{\beta q}\left(x_{i}\right)-w_{\beta}\left(x_{i}\right) w_{q}\left(x_{i}\right)}{\left[w\left(x_{i}\right)\right]^{2}}, \\
& \mathcal{L}_{22}=\frac{\partial^{2} \mathcal{L}}{\partial \beta^{2}}=\sum_{i=1}^{n} \frac{w\left(x_{i}\right) w_{\beta \beta}\left(x_{i}\right)-\left[w_{\beta}\left(x_{i}\right)\right]^{2}}{\left[w\left(x_{i}\right)\right]^{2}},
\end{aligned}
$$

where $w_{q}(x)=1 / q\left[r(x-1) e^{(x-1)^{\beta}}-r(x) e^{x^{\beta}}\right]$,

$$
\begin{aligned}
w_{\beta}(x)= & {\left[r(x-1)(x-1)^{\beta} \ln (x-1) e^{(x-1)^{\beta}}-r(x) x^{\beta} \ln (x) e^{x^{\beta}}\right] \ln q, } \\
w_{q q}(x)= & \frac{1}{q^{2}}\left\{r(x-1) e^{(x-1)^{\beta}}\left[r(x-1) e^{(x-1)^{\beta}}-1\right]-r(x) e^{x^{\beta}}\left[r(x) e^{x^{\beta}}-1\right]\right\}, \\
w_{q \beta}(x)= & \frac{\ln q}{q}\left[r(x-1) e^{2(x-1)^{\beta}} \ln (x-1)-r(x) e^{2 x^{\beta}} \ln x+\frac{w_{\beta}(x)}{\ln q}\right], \\
w_{\beta \beta}(x)= & r(x-1)(x-1)^{\beta}(\ln (x-1))^{2} e^{(x-1)^{\beta}} \ln q\left[1+(x-1)^{\beta}\left(1+e^{(x-1)^{\beta}} \ln q\right)\right] \\
& -r(x) x^{\beta}(\ln x)^{2} e^{x^{\beta}} \ln q\left[1+x^{\beta}\left(1+e^{x^{\beta}} \ln q\right)\right] .
\end{aligned}
$$

The observed information matrix is a two-by-two symmetric matrix

$$
\mathfrak{I}(q, \beta)=-\left[\begin{array}{ll}
\mathcal{L}_{11} & \mathcal{L}_{12} \\
\mathcal{L}_{21} & \mathcal{L}_{22}
\end{array}\right] .
$$

For a relative maximum of $\mathcal{L}$, which occurs at the MLE of the parameters $q$ and $\beta$, the information matrix $\mathfrak{I}(\hat{q}, \hat{\beta})$ must be positive definite. It is known that the $\operatorname{MLE}$ of $(q, \beta)$ approximately follows 
Table 1: List of survival functions of the models used here

\begin{tabular}{lcl}
\hline \hline \multicolumn{1}{c}{ Model } & $S(x)$ & \multicolumn{1}{c}{ Reference } \\
\hline $\operatorname{DW}(q, \beta)$ & $q^{x^{\beta}}$ & Nakagawa and Osaki (1975) \\
$\operatorname{DMW}(q, \beta)$ & $q^{x^{\beta} \alpha^{x}}$ & Nooghabi et al. $($ 2012) \\
$\operatorname{DAddW}(q, \beta, \theta, \gamma)$ & $q^{x^{\beta}+\theta x^{\gamma}}$ & Bebbington et al. $(2012)$ \\
$\operatorname{DRMW}(q, b, c)$ & $q^{\sqrt{x}\left(1+b c^{x}\right)}$ & Almalki and Nadarajah (2014) \\
\hline \hline DW = discre Weibull; DMW = discrete modified Weibull; DAddW = discrete additive Weibull; DRMW = discrete reduced
\end{tabular}
modified Weibull.

normal distribution with mean of $(q, \beta)$ and variance covariance matrix equals the inverse of $\Im(\hat{q}, \hat{\beta})$. Using this result, one can calculate the large-sample $(1-\alpha) 100 \%$ confidence intervals for $q$ and $\beta$.

\subsection{Bootstrap intervals}

We use bootstrap method to approximate the distribution of the point estimators of the parameters. Then, we can use the bootstrap distribution to estimate the confidence intervals. Let $\hat{\theta}=\theta\left(X_{1}, \ldots, X_{n}\right)$ be a point estimator of the parameter of interest $\theta$ using a give data set $X_{1}, \ldots, X_{n}$. The bootstrap is a method to estimate the distribution of the statistic $\hat{\theta}$ by getting random sample $\theta_{1}^{*} \theta_{2}^{*}, \ldots, \theta_{B}^{*}$ for $\theta$ based on $B$ random samples that are drawn with replacement from the original data $X_{1}, X_{2}, \ldots, X_{n}$, see Wasserman (2006). The bootstrap sample $\theta_{1}^{*} \theta_{2}^{*}, \ldots, \theta_{B}^{*}$ can be used to construct bootstrap confidence intervals. We will use the normal, pivotal and percentile intervals, which are the easiest and most accurate methods to calculate the intervals. We use the quartile, least square and maximum likelihood methods to derive the bootstrap samples $\theta_{1}^{*} \theta_{2}^{*}, \ldots, \theta_{B}^{*}$.

- Bootstrap normal interval: The bootstrap normal confidence interval (BNCI) is the simplest bootstrap interval. A $(1-\alpha) 100 \%$ BNCI of $\theta$ is given by

$$
\hat{\theta} \pm z_{\frac{\alpha}{2}} \hat{\mathbf{s e}}_{\text {boot }} \text {, where } \quad \hat{\mathbf{s e}}_{\text {boot }}=\sqrt{\frac{1}{B} \sum_{b=1}^{B}\left(\theta_{b}^{*}-\frac{1}{B} \sum_{b=1}^{B} \theta_{b}^{*}\right)^{2}} .
$$

This interval is not accurate unless the distribution of $\hat{\theta}$ is close to normal.

- Bootstrap pivotal interval: The $(1-\alpha) 100 \%$ bootstrap pivotal confidence interval (BPTCI) of a parameter $\theta$ is $\left(2 \hat{\theta}-\theta_{B(1-\alpha / 2)}^{*}, \quad 2 \hat{\theta}-\theta_{B \alpha / 2}^{*}\right)$, where $\theta_{B \alpha / 2}^{*}$ and $\theta_{B(1-\alpha / 2)}^{*}$ are the $\alpha / 2$ and $1-\alpha / 2$ quantiles of the bootstrap sample.

- Bootstrap percentile interval: The $(1-\alpha) 100 \%$ bootstrap percentile confidence interval (BPCI) of a parameter $\theta$ is defined by $\left(\theta_{B \alpha / 2}^{*}, \theta_{B(1-\alpha / 2)}^{*}\right)$, where $\theta_{B \alpha / 2}^{*}$ and $\theta_{B(1-\alpha / 2)}^{*}$ are the $\alpha / 2$ and $1-\alpha / 2$ quantiles of the bootstrap sample.

\section{Data analysis}

In this section, we compare the results for the fit of the proposed distribution (DTPBT) with discrete Weibull (DW), discrete modified Weibull (DMW), discrete additive Weibull (DAddW) and discrete reduced modified Weibull (DRMW) using two real data sets. Table 1 lists the survival functions and references for these distributions.

Since these distributions are not of the same family of the proposed distribution, we use the Bayesian Information Criterion (BIC) of Schwarz (1978) to compare their goodness of fit. BIC is 
Table 2: The MLE, se, and BIC for the five models

\begin{tabular}{|c|c|c|c|c|c|c|}
\hline & Model & & & $\mathrm{LE}$ & & BIC \\
\hline \multirow{5}{*}{ Aarset data } & DTPBT & $\begin{array}{c}\hat{q}=0.9758 \\
\left(9.542 \times 10^{-3}\right)\end{array}$ & $\begin{array}{c}\hat{\beta}=0.3352 \\
(0.0223)\end{array}$ & & & 467.5 \\
\hline & DMW & $\begin{array}{c}\hat{q}=0.9403 \\
(0.02600)\end{array}$ & $\begin{array}{c}\hat{\beta}=0.345 \\
(0.1260)\end{array}$ & $\begin{array}{c}\hat{\alpha}=1.0241 \\
(0.0050)\end{array}$ & & 469.9 \\
\hline & DAddW & $\begin{array}{c}\hat{q}=0.9216 \\
(0.03400)\end{array}$ & $\begin{array}{c}\hat{\beta}=0.4541 \\
(0.1190)\end{array}$ & $\begin{array}{c}\hat{\theta}=6.0091 \times 10^{-5} \\
\left(2.181 \times 10^{-4}\right)\end{array}$ & $\begin{array}{c}\hat{\gamma}=2.8387 \\
(0.807)\end{array}$ & 472.1 \\
\hline & DW & $\begin{array}{c}\hat{q}=0.9805 \\
(0.01100)\end{array}$ & $\begin{array}{c}\hat{\beta}=1.0234 \\
(0.1310)\end{array}$ & & & 491.0 \\
\hline & DRMW & $\begin{array}{l}\hat{q}=0.847 \\
(0.01998)\end{array}$ & $\begin{array}{c}\hat{b}=1.109 \\
(0.7488)\end{array}$ & $\begin{array}{c}\hat{c}=0.621 \\
(0.1772)\end{array}$ & & 503.5 \\
\hline \multirow{5}{*}{ Devices data } & DTPBT & $\begin{array}{c}\hat{q}=0.9899 \\
(0.00670)\end{array}$ & $\begin{array}{c}\hat{\beta}=0.2887 \\
(0.0223)\end{array}$ & & & 225.0 \\
\hline & DMW & $\begin{array}{c}\hat{q}=0.9465 \\
(0.06300)\end{array}$ & $\begin{array}{c}\hat{\beta}=0.3194 \\
(0.2540)\end{array}$ & $\begin{array}{c}\hat{\alpha}=1.0055 \\
\left(1.905 \times 10^{-3}\right)\end{array}$ & & 228.3 \\
\hline & DAddW & $\begin{array}{c}\hat{q}=0.9771 \\
(0.02700)\end{array}$ & $\begin{array}{c}\hat{\beta}=0.5208 \\
(0.3464)\end{array}$ & $\begin{aligned} \hat{\theta}= & 4.015 \times 10^{-3} \\
& (0.0187)\end{aligned}$ & $\begin{array}{c}\hat{\gamma}=1.677 \\
(0.764)\end{array}$ & 231.5 \\
\hline & DW & $\begin{array}{c}\hat{q}=0.9912 \\
\left(9.257 \times 10^{-3}\right)\end{array}$ & $\begin{array}{c}\hat{\beta}=1.0234 \\
(0.1890)\end{array}$ & & & 227.9 \\
\hline & DRMW & $\hat{q}=0.9191$ & $\hat{b}=0.0364$ & $\hat{c}=0.0581$ & & 241.9 \\
\hline
\end{tabular}

DTPBT = discrete two-parameter bathtub; DMW = discrete modified Weibull; DAddW = discrete additive Weibull; DW = discrete Weibull; DRMW = discrete reduced modified Weibull; MLE = maximum likelihood estimates; se = standard error; $\mathrm{BIC}=$ Bayesian information criterion.

defined as BIC $=-2 \mathcal{L}+k \ln n$, where $\mathcal{L}$ is the value of $\log$-likelihood function evaluated at the MLE of the model's parameters, $n$ is the sample size and $k$ is the number of the model's parameters.

- Aarset data: Aarset (1987) data consist of the failure times (in weeks) of 50 devices put on a life test. The TTT-plot for this data shows that the hazard rate has a bathtub-shape. Nooghabi et al. (2012) used the DMW distribution, Bebbington et al. (2012) used DAddW, and Almalki and Nadarajah (2014) used DRMW to analyze this data and reported that all of these distributions fit the data well.

- Electronic devices data: In this application, 18 electronic devices were put on a life test and their lifetimes (in days) were observed as 5, 11, 21, 31, 46, 75, 98, 122, 145, 165, 196, 224, 245, 293, $321,330,350$, 420. Wang (2000) showed that this data have a bathtub hazard shape and used the additive Burr XII distribution to analyze it.

We use the proposed model to re-analyze these two data sets and compare it with the models mentioned above. Table 2 shows the MLE of the two-parameters, the corresponding standard error (se) and BIC for all models, for both two data sets. Based on the BIC values, we conclude that the DTPBT distribution fits both data sets better than all those distributions listed here. To show the existence and uniqueness of the MLE, we draw the log relative likelihood functions for parameters (Figure 4). Figure 5 displays the likelihood regions for $(q, \beta)$ using the two data sets. The QEs of $(q, \beta)$ are $(0.9570,0.2832)$ and $(0.9769,0.2484)$ for Aarset and devices data, respectively. The LSE of $(q, \beta)$ are $(0.9718,0.3119)$ for Aarset data and $(0.9851,0.2727)$ for devices data. Table 3 gives the asymptotic $95 \%$ confidence interval and the $14.7 \%$ likelihood interval for $(q, \beta)$ and their corresponding widths for both two data sets. Based on the widths of the intervals, as expected, we conclude that the likelihood interval provides a better approximate than the asymptotic technique. 

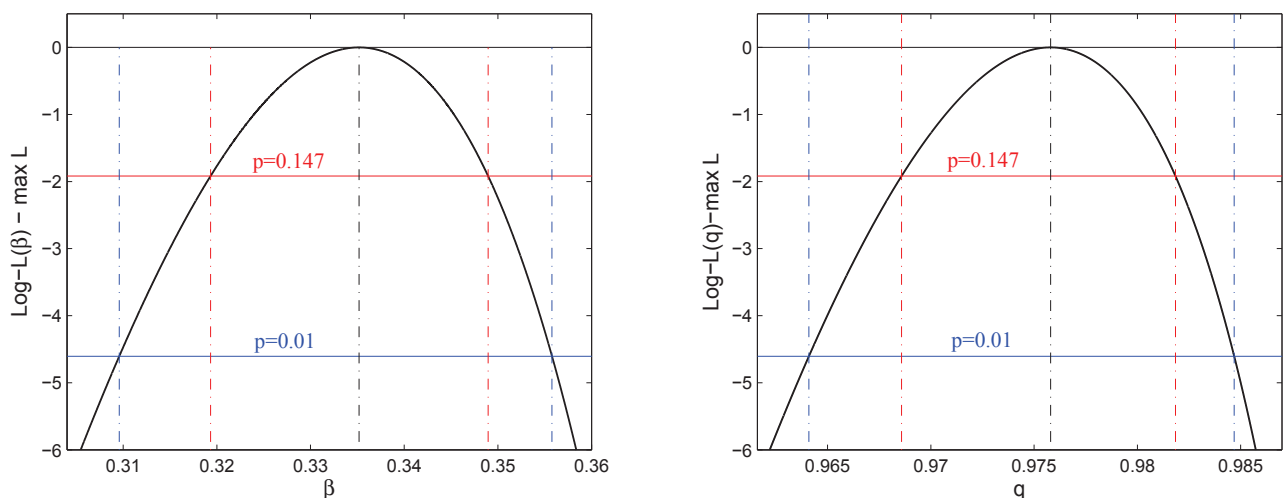

Figure 4: $\log$ relative likelihood functions for $\beta$ and $q$ along with the $1 \%$ and $14.7 \%$ likelihood intervals using Aarset data.
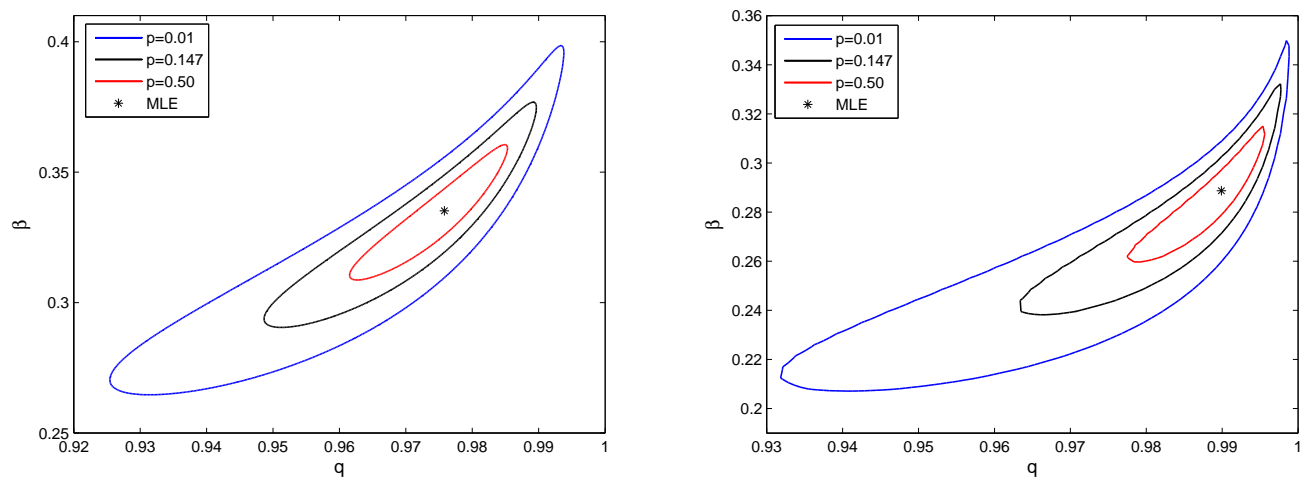

Figure 5: The likelihood regions for $(q, \beta)$ using the Aarset data (left) and devices data (right).

Table 3: The asymptotic CI and LI and corresponding widths

\begin{tabular}{ccccccc}
\hline \hline \multirow{2}{*}{ Method } & \multicolumn{2}{c}{$q$} & & \multicolumn{2}{c}{$\beta$} \\
\cline { 3 - 4 } \cline { 6 - 7 } & & Interval & Width & & Interval & Width \\
\hline \multirow{2}{*}{ Aarset data } & Asymptotic & $(0.9571,0.9945)$ & 0.0374 & & $(0.2934,0.3769)$ & 0.0835 \\
& LI & $(0.9686,0.9818)$ & 0.0133 & & $(0.3193,0.3489)$ & 0.0296 \\
\hline \multirow{2}{*}{ Devices data } & Asymptotic & $(0.9768,1.0000)$ & 0.0232 & & $(0.2450,0.3324)$ & 0.0874 \\
& LI & $(0.9845,0.9939)$ & 0.0093 & & $(0.2713,0.3025)$ & 0.0313 \\
\hline \hline
\end{tabular}

$\mathrm{LI}=$ likelihood interval.

The mean, median of the bootstrap distribution of the point estimators of the DTPBT model and the bootstrap confidence intervals for the two data sets using the three different estimation techniques discussed are given in Table 4. In order to compare the three techniques used to estimate the DTPBT model's parameters, we calculated the Kolmogorov-Smirnov (KS) test statistic, along with the corresponding $p$-value, for every technique using the three data sets (Table 5). Based on the $p$-value, one can see that the least squares and quartile technique provide point estimates which led to a rejection of the DTPBT distribution to fit the Aarset data while the maximum likelihood method does not. For the devices data, all methods provide estimates that led to not reject DTPBT model. However, based 
Table 4: The mean and median of the bootstrap distribution of the point estimators and the bootstrap CIs

\begin{tabular}{|c|c|c|c|c|c|c|c|}
\hline & \multirow{2}{*}{ Method } & \multirow{2}{*}{ Par } & \multirow[b]{2}{*}{ Mean } & \multirow[b]{2}{*}{ Median } & \multicolumn{3}{|c|}{ Bootstrap } \\
\hline & & & & & $\mathrm{BNCI}$ & BPTCI & BPCI \\
\hline \multirow{6}{*}{ Aarset data } & \multirow{2}{*}{$\mathrm{QE}$} & $q$ & 0.9512 & 0.9582 & $(0.8962,1.0178)$ & $(0.9333,1.0387)$ & $(0.8754,0.9807)$ \\
\hline & & $\beta$ & 0.2878 & 0.2925 & $(0.2055,0.3608)$ & $(0.2155,0.3635)$ & $(0.2028,0.3508)$ \\
\hline & \multirow{2}{*}{ LS } & $q$ & 0.9629 & 0.9679 & $(0.9003,1.0143)$ & $(0.9183,1.0274)$ & $(0.8872,0.9963)$ \\
\hline & & $\beta$ & 0.3092 & 0.3081 & $(0.1729,0.3881)$ & $(0.1396,0.3675)$ & $(0.1935,0.4214)$ \\
\hline & \multirow{2}{*}{ ML } & $q$ & 0.9722 & 0.9752 & $(0.9452,1.0060)$ & $(0.9576,1.0171)$ & $(0.9345,0.9940)$ \\
\hline & & $\beta$ & 0.3358 & 0.3356 & $(0.2783,0.3920)$ & $(0.2746,0.3870)$ & $(0.2833,0.3958)$ \\
\hline \multirow{6}{*}{ Devices data } & \multirow{2}{*}{ QE } & $q$ & 0.9770 & 0.9804 & $(0.9434,1.0104)$ & $(0.9546,1.0209)$ & $(0.9329,0.9992)$ \\
\hline & & $\beta$ & 0.2685 & 0.2636 & $(0.1656,0.3312)$ & $(0.1289,0.2911)$ & $(0.2056,0.3679)$ \\
\hline & \multirow{2}{*}{ LS } & $q$ & 0.9803 & 0.9837 & $(0.9464,1.0238)$ & $(0.9706,1.0305)$ & $(0.9396,0.9995)$ \\
\hline & & $\beta$ & 0.2820 & 0.2766 & $(0.1803,0.3650)$ & $(0.1644,0.3451)$ & $(0.2003,0.3809)$ \\
\hline & \multirow{2}{*}{ ML } & & 0.9889 & 0.9909 & $(0.9743,1.0056)$ & $(0.9810,1.0100)$ & $(0.9699,0.9989)$ \\
\hline & & $\beta$ & 0.2991 & 0.2970 & $(0.2417,0.3361)$ & $(0.2260,0.3212)$ & $(0.2566,0.3518)$ \\
\hline
\end{tabular}

$\mathrm{QE}=$ quartile estimate; $\mathrm{LS}$ = least squares; $\mathrm{ML}$ = maximum likelihood; $\mathrm{BNCI}=$ bootstrap normal confidence interval;

$\mathrm{BPTCI}=$ bootstrap pivotal confidence interval; $\mathrm{BPCI}=$ bootstrap percentile confidence interval.

Table 5: The Kolmogorov-Smirnov values and the corresponding $p$-values

\begin{tabular}{cccc}
\hline \hline Data & MLE & LSM & Quartile \\
\hline Aarset & $0.1508(0.1856)$ & $0.2059(0.0246)$ & $0.2333(0.0071)$ \\
Devices & $0.0981(0.9881)$ & $0.0761(0.9997)$ & $0.1322(0.8717)$ \\
\hline \hline
\end{tabular}

MLE = maximum likelihood estimates; LSM = least squares method.

on the KS test, the least squares method provides slightly better estimates than the MLE.

\section{Simulation study}

A simulation study is conducted to investigate the performance of the accuracy of three estimation methods used. The following scheme is applied:

1. Specify the values of the parameters $q$ and $\beta$

2. Specify the sample size $n$

3. Generate a random sample with size $n$ from $\operatorname{DTPBT}(q, \beta)$

4. Using every estimation method used in this paper

(a) Calculate the point estimates of the two-parameters $q$ and $\beta$

(b) Calculate the squared deviation of the point estimate from the exact value of each parameter

5. Repeat steps 3-4, $N$ times

6. Calculate the average of the point estimates (APE) and the mean squared error (MSE) for each parameter using every estimation method as discussed

The simulation study is performed when $N=1000$, at different sample sizes, and the parameter values $(q, \beta)=(0.9,0.2),(0.9,0.7)$, and $(0.9,1.1)$. The selected values of $(q, \beta)$ provide different hazard shapes. Table 6 displays the APEs and MSEs for each parameter. There are some cases in which the quartile method fails. This might happen when the $1^{\text {st }}$ and $3^{r d}$ quartiles are equal. The results in Table 
Table 6: The MSEs ( $1^{\text {st }}$ row) and the average of point estimates ( $2^{\text {nd }}$ row) of $q$ and $\beta$ using the three methods

\begin{tabular}{|c|c|c|c|c|c|c|c|}
\hline \multirow{2}{*}{$(q, \beta)$} & \multirow{2}{*}{$n$} & \multicolumn{2}{|c|}{ Maximum likelihood } & \multicolumn{2}{|c|}{ Least squares } & \multicolumn{2}{|c|}{ Quartile } \\
\hline & & $q$ & $\beta$ & $q$ & $\beta$ & $q$ & $\beta$ \\
\hline \multirow{8}{*}{$(0.9,0.2)$} & \multirow{2}{*}{10} & 0.00362 & 0.00180 & 0.00235 & 0.01383 & 0.00218 & 0.00344 \\
\hline & & 0.90083 & 0.21646 & 0.93539 & 0.27640 & 0.90874 & 0.22471 \\
\hline & \multirow{2}{*}{40} & 0.00082 & 0.00031 & 0.00149 & 0.00414 & 0.00087 & 0.00075 \\
\hline & & 0.90123 & 0.20449 & 0.92614 & 0.23162 & 0.90556 & 0.20879 \\
\hline & \multirow{2}{*}{100} & 0.00030 & 0.00011 & 0.00129 & 0.00235 & 0.00041 & 0.00030 \\
\hline & & 0.90077 & 0.20203 & 0.92325 & 0.22298 & 0.90596 & 0.20654 \\
\hline & \multirow{2}{*}{145} & 0.00023 & $7.55 e-05$ & 0.00106 & 0.00178 & 0.00032 & 0.00023 \\
\hline & & 0.89987 & 0.20112 & 0.92280 & 0.22059 & 0.90441 & 0.20479 \\
\hline \multirow{8}{*}{$(0.9,0.7)$} & \multirow{2}{*}{10} & 0.00312 & 0.02432 & 0.00326 & 0.14204 & $\mathrm{NaN}$ & $\mathrm{NaN}$ \\
\hline & & 0.90515 & 0.77106 & 0.95014 & 0.97668 & $\mathrm{NaN}$ & $\mathrm{NaN}$ \\
\hline & \multirow{2}{*}{40} & 0.00074 & 0.00394 & 0.00232 & 0.04827 & 0.00194 & 0.01697 \\
\hline & & 0.90315 & 0.71733 & 0.94453 & 0.86767 & 0.93620 & 0.76616 \\
\hline & \multirow{2}{*}{100} & 0.00030 & 0.00143 & 0.00210 & 0.03496 & 0.00151 & 0.01039 \\
\hline & & 0.90119 & 0.70710 & 0.94265 & 0.85066 & 0.93680 & 0.75934 \\
\hline & \multirow{2}{*}{145} & 0.00023 & 0.00098 & 0.00197 & 0.03608 & 0.00147 & 0.01012 \\
\hline & & 0.89998 & 0.70407 & 0.94130 & 0.84614 & 0.93684 & 0.76109 \\
\hline \multirow{8}{*}{$(0.9,1.1)$} & \multirow{2}{*}{10} & 0.00451 & 0.66628 & 0.00475 & 0.84837 & $\mathrm{NaN}$ & $\mathrm{NaN}$ \\
\hline & & 0.90206 & 1.37379 & 0.96431 & 1.73713 & $\mathrm{NaN}$ & $\mathrm{NaN}$ \\
\hline & \multirow{2}{*}{40} & 0.00094 & 0.01788 & 0.00500 & 0.51887 & 0.00453 & 0.02166 \\
\hline & & 0.90265 & 1.13795 & 0.96889 & 1.74157 & 0.96127 & 1.18716 \\
\hline & \multirow{2}{*}{100} & 0.00038 & 0.00468 & 0.00487 & 0.45942 & 0.00479 & 0.01593 \\
\hline & & 0.89977 & 1.10759 & 0.96851 & 1.71742 & 0.96792 & 1.21413 \\
\hline & \multirow{2}{*}{145} & 0.00024 & 0.00290 & 0.00487 & 0.42921 & 0.00482 & 0.01489 \\
\hline & & 0.90016 & 1.10585 & 0.96871 & 1.71207 & 0.96907 & 1.21885 \\
\hline
\end{tabular}

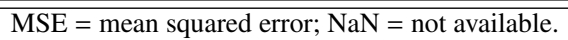

6 indicate that: (1) the MSE associated with the MLE decrease as the sample size increases, (2) MSEs associated with both the least squares and quartile methods do not decrease monotonically with the sample size, (3) quartile estimates are not available in some situations (mostly at low sample sizes and for some values of the parameters), (4) the quartile estimation and least squares method may lead to the overfitting problem, (5) the MSEs associated with the MLE are smaller than that associated with the least squares and quartile estimates.

\section{Conclusion}

A new discrete two-parameter distribution is introduced and studied in this paper. The model provides more flexibility than other two-parameter discrete distributions in terms of hazard rate function shape. The proposed distribution is the only two-parameter discrete distribution that displays a bathtub hazard shape. An easy formula to generating random samples from the proposed distribution is given. Three different classical techniques (maximum likelihood, least squares and quartile) are applied to estimate the two-parameters of the distribution. Different approaches are also used to discuss the confidence intervals for the two-parameters. Application of the proposed distribution to two real data sets show a better fit for the proposed distribution than other well-known discrete distributions. A simulation study is conducted to examine the accuracy of the three different methods of the model parameter. 


\section{References}

Aarset MV (1987). How to identify a bathtub hazard rate, IEEE Transactions on Reliability, R-36, 106-108.

Al-Huniti AA and Al-Dayian GR (2012). Discrete Burr type III distribution, American Journal of Mathematics and Statistics, 2, 145-152.

Almalki SJ (2013). A reduced new modified Weibull distribution, Retrieved January 17, 2017, from: http://arxiv.org/abs/1307.3925

Almalki SJ and Nadarajah S (2014). A new discrete modified Weibull distribution, IEEE Transactions on Reliability, 63, 68-80.

Bebbington M, Lai CD, Wellington M, and Zitikis R (2012). The discrete additive Weibull distribution: a bathtub-shaped hazard for discontinuous failure data, Reliability Engineering and System Safety, 106, 37-44.

Chen Z (2000). A new two-parameter lifetime distribution with bathtub shape or increasing failure rate function, Statistics \& Probability Letters, 49, 155-161.

Gnedenko BV and Ushakov IA (1995). Probabilistic Reliability Engineering, John Wiley \& Sons, New York.

Kalbfleisch JG (1985). Probability and Statistical Inference (Volume 2: Statistical Inference), Springer -Verlag, New York.

Kapur KC and Lamberson LR (1977). Reliability and Engineering Design, John Wiley \& Sons, New York.

Lai CD, Xie M, and Murthy DNP (2003). A modified Weibull distribution, IEEE Transactions on Reliability, 25, 33-37.

Lawless JF (1982). Statistical Models and Methods for Lifetime Data, John Wiley \& Sons, New York.

Nakagawa T and Osaki S (1975). The discrete Weibull distribution, IEEE Transactions on Reliability, R-24, 300-301.

Nooghabi MS, Borzadaran GRM, and Roknabadi AHR (2012). Discrete modified Weibull distribution, Metron, 69, 207-222.

Padgett WJ and Spurrier JD (1985). On discrete failure models, IEEE Transactions on Reliability, 34, $253-256$.

Roy D (2003). The discrete normal distribution, Communications in Statistics - Theory and Methods, 32, 1871-1883.

Roy D (2004). Discrete Rayleigh distribution, IEEE Transactions on Reliability, 53, 255-260.

Schwarz G (1978). Estimating the dimension of a model, Annals of Statistics, 6, 461-464.

Sinha SK (1986). Reliability and Life Testing, John Wiley \& Sons, New York.

Stein WE and Dattero R (1984). A new discrete Weibull distribution, IEEE Transactions on Reliability, 33, 196-197.

Wang FK (2000). A new model with bathtub-shaped failure rate using an additive Burr XII distribution, Reliability Engineering and System Safety, 70, 305-312.

Wasserman L (2006). All of Nonparametric Statistics, Springer, New York.

Xie M and Lai CD (1996). Reliability analysis using an additive Weibull model with bathtub-shaped failure rate function, Reliability Engineering and System Safety, 52, 87-93. 